\title{
Improvement of Health Sector in Kenya
}

\author{
Haradhan Kumar Mohajan* \\ Assistant Professor, Premier University, Chittagong, Bangladesh \\ *Corresponding author: haradhan1971@gmail.com
}

Received July 29, 2014; Revised August 10, 2014; Accepted August 18, 2014

\begin{abstract}
Kenya faces major socio-economic and health challenges since the independent 1963. Tuberculosis, HIV/AIDS and malaria are the leading causes of morbidity and mortality in Kenya. The Government of Kenya has been trying to build a health system which can effectively provide quality health services to the population of the country. In Kenya, health services are provided through a network of over 5,000 health facilities countrywide. The country spends $5.1 \%$ of its GDP on health sector. As a result both mortality and morbidity rates are decreasing in Kenya. Infant and under-5 mortality rates are high in the country compare to the some other developing countries. Despite many attempts are taken by the Government yet the health sector of Kenya is far to reach the Millennium Development Goals. The paper discusses aspects of health of Kenya to make a healthy nation in the world.
\end{abstract}

Keywords: health sector, Malaria, TB, HIV/AIDS, ART, Kenya

Cite This Article: Haradhan Kumar Mohajan, "Improvement of Health Sector in Kenya.” American Journal of Public Health Research, vol. 2, no. 4 (2014): 159-169. doi: 10.12691/ajphr-2-4-6.

\section{Introduction}

Republic of Kenya is the $4^{\text {th }}$ largest economy in SubSaharan Africa (SSA) and Nairobi is its capital city. Mombasa is the main sea port of the country. Kenya is named after a mountain (5,199 $\mathrm{m}$ above sea level) of the same name of the country. Its area is $580,400 \mathrm{~km}^{2}$, which is $49^{\text {th }}$ largest country in the world and water area is $11,227 \mathrm{~km}^{2}$ (2.3\% of the total area), $80 \%$ of total land area is arid or semi-arid and forest area is $6 \%$ of the total land. It is the $6^{\text {th }}$ most populated country in SSA and $31^{\text {st }}$ largest populated country in the world with estimated 45 million inhabitants in 2014 (of which about 75-80\% lives in rural areas), with a majority under- 15 . The density of population in Kenya is 69 persons $/ \mathrm{km}^{2}$, which is the $140^{\text {th }}$ densely populated country in the world [28].

Kenya lies between latitudes $5^{\circ} \mathrm{N}$ and $5^{\circ} \mathrm{S}$ and between longitudes $34^{\circ} \mathrm{E}$ and $42^{\circ} \mathrm{E}$. It is almost bisected by the equator. The country is divided into seven ecological zones: Tropical Alpine, Upper Highland, Lower Highland, Upper Midland, Lower Midland, Lowland and Coastal Lowland [11].

More than $60 \%$ people of Kenya live below the poverty line (less than $\$ 1.25$ a day or unable to afford to buy food providing a daily intake of 2,100 kilocalories). Like other SSA countries, Kenya faces major socio-economic and health challenges. Kenya signed in the global Millennium Development Goals (MDGs) in 2000 to guide prioritization of countries as they move towards improvement of their development. The country also has specifically defined its long term Vision, the Vision 2030 that elaborates what the country intends to focus on as it moves towards a medium income country by 2030 [9].
The Government of Kenya (GoK) has been trying to build a health system which can effectively provide quality health services to the population of the country. Life expectancy (at birth) of the total population in 2013 was about 63.29 years (male 61.84 years and female 64.77 years) compared to a global average of 68 years. Loss of healthy life-years (about 48 years) is about $82 \%$ due to infectious disease. The persistence of malaria, tuberculosis and the progression of the HIV/AIDS epidemic have contributed to the fall in life expectancy and the rise in mortality rates, particularly for infants and under-5 [8].

A new Kenya Health Policy (2012-2030) was adopted by the Government in 2012. It is associated to the 2010 constitution and the country's long-term development agenda. The health policy embraces this long-term vision and has made use of the primary health centre (PHC) approach to focus on the attainment of the right to health for all citizens as provided for in the constitution. A Government-wide medium-term development plan (MTP) is at advanced stage of development. Alongside the MTP is a National Health Strategic Plan (NHSSP III) also at advanced stage of development [66].

In Kenya infant mortality rate is about 58.1 per 1,000 live births. The overall under- 5 child mortality rate is about 121 per 1,000 live births, which is double of the global average and maternal mortality rate is about 414 per 1,000 [9]. Kenya has high morbidity and mortality rates affecting the population of all ages, especially children under-5. A significant proportion of this morbidity and mortality is due to infectious conditions, but many other non-infectious factors are chronic diseases and economic deprivation resulting in poverty, malnutrition and inadequate health care. About 15 women die every day due to pregnancy related complications in Kenya and $20 \%$ of all deaths among mothers in the country are AIDS related [20]. 
It has been estimated that about 200 people die of the disease in Kenya every day and all these deaths are preventable. Tuberculosis, Human immunodeficiency virus (HIV)/Acquired immune deficiency syndrome (AIDS) and malaria are the leading causes of morbidity and mortality in Kenya [66].

Malaria is a leading cause of childhood morbidity and mortality worldwide. It is estimated that about 732,000 deaths of world among children under-5 is due to malaria, among them Africa accounts 16\% [3]. Kenya adopted a new National Malaria Strategy (NMS) in 2001, which emphasized the distribution of insecticide treated bed nets (ITNs) for short in line and recommended selective indoor residual spraying (IRS) for epidemic control and prompt treatment, and prophylactic treatment. Kenya is working towards achieving the Abuja targets of $80 \%$ ITN coverage. Malaria has been eradicated from most of the countries of the world, but it remains threats to many people of Africa. About $80 \%$ of the world's malaria cases and $90 \%$ of malaria-related deaths occur in SSA. In 2010, there were 174 million estimated cases of malaria on the African and about 600,000 deaths are recorded. In Kenya about 70\% of the population is at risk from malaria, which kills an estimated 34,000 children under-5 each year [1].

Kenya is one of the 22 high TB burden countries of the world. According to the WHO Global Tuberculosis Report 2010 Kenya is $13^{\text {th }}$ ranked. Kenya reported more than 106,083 TB cases in 2010 with more than 4,000 deaths. About 297 Health professionals are deployed by the GoK to be engaged in TB control programme including 46 at national, 36 at provincial and 215 at district level technically reporting to Division of Leprosy, TB, and other lung disease (DLTLD) [65].

In Kenya about $7 \%$ of adults, and $6 \%$ of rural residents, are infected with HIV. Rates of infection amongst women are almost twice as high as those of men (8.7\% to $4.6 \%)$, which reflect the particular vulnerability of young women to HIV infection compared with young men. In Kenya 1 child in 9 is orphaned. About 9\% of children under-15 have lost their fathers, $4 \%$ have lost their mothers, and $2 \%$ have lost both of their parents. Only $58 \%$ of children live with both parents [5]. In 2003, there were 650,000 AIDS orphans in Kenya. In 2008, the Government began providing free antiretroviral drugs (ARVs) to HIVpositive people who need them, and medical care is now free for children under-5 [44].

Children are also vulnerable to diarrhea and pneumonia. There was unreliable evidence of a rise in typhoid in several areas, due to increasingly difficult access to clean potable water, poor sanitation and overcrowded housing conditions. It is hard to find clean water, because good wells are miles from the home. Many parents have no concept of what a balanced diet is [18].

In Kenya, $12 \%$ of the population is obese and most of them are found in urban areas. In Kenya obesity is considered a sign of wealth. In real life obesity is a major disease which creates Type 2 diabetes and cardiac attack. Obesity has increased along with Type 2 diabetes in the urban areas of Kenya. If a person is slim others will consider him as a HIV/AIDS infected person. For this reason AIDS infected people took more food to show others that they are not infected by this fatal disease. Additionally in Kenya fast food availability has increased in the cities. Cities are developed without the scope of the facilities of exercise [27].

Childhood vaccination coverage in Kenya has declined, with a fall of the proportion of children aged 12-23 months fully vaccinated from $79 \%$ in 1993 to $52 \%$ in 2003 [13]. Infant and under-5 mortality rates are increasing, from $73 \%$ and $105 \%$ respectively in 1989 1993 to $77 \%$ and $115 \%$ in 2003 . The under-5 mortality rate is higher in rural areas. The rates vary greatly by province, and according to mothers' level of education. Except for neonatal mortality, all childhood mortality indicators were highest in Nyanza Province and lowest in the Central Province [6]. The level of maternal mortality is very high in Kenya [54].

The patients expect high quality of services in hospitals and other health care centers. Quality in healthcare may comprise of newer technology, newer and effective medication, and higher staff to patient ratios, affordability, efficiency and effectiveness of service delivery [47].

Total expenditure on healthcare increased from \$219 million in 2005 to $\$ 277.5$ million in 2007, and between 2004 and 2008 the number of health institutions increased by $19.8 \%$ in Kenya [44].

We have analyzed the secondary data to prepare the article. The data are collected from the annual reports of Ministry of Health of Kenya, Country Profiles of Kenya, Global and Kenyan WHO reports on health and diseases, various research articles, and websites on health.

\section{Background of Health Policy of Kenya}

Health is defined being not only the absence of disease but also general mental, physical, and social well-being. Good health is renowned as a vital pillar in ensuring the prosperity of individual, household, community of a country. People seek good health to maximize their health stock, to ensure they are able to carry out the activities that will improve their overall social welfare. All persons in a society are responsible for creating the conditions that ensure the health stock available is maximized [17].

Kenya's health policy has been based on the country's landmark post-colonial nation-building and socioeconomic development [59]. Since independence the GoK has given high priority to the improvement of the health sector of the country [20]. In the 1970s health indicators in Kenya deteriorated from the mid-1980s with the growing population and worsening socio-economic and political environment, and a severe social development crisis occurred in the 1990s [30]. Since 1994, the health sector development agenda has been guided by the Kenya Health Policy Framework Paper (KHPFP) up to 2010. The economy grew to about $6 \%$ in 2006 and healthcare reforms were intensified in an attempt to reduce the household burden of accessing primary health services.

In Kenya death rate has declined from 20 per 1,000 person in 1963 to 13 in 1987, and 12 in 1991. Life expectancy increased from 40 to 58 years in 1960 and 1994; infant mortality declined from 126 per 1000 in 1962 to 60 per 1000 in 1994, and the immunization coverage rose to $70 \%$ in 1994 from less than $40 \%$ at independence in 1963 [34].

In 1994, the Government of Kenya (GoK) approved the Kenya Health Policy Framework (KHPF) as a blueprint 
for developing and managing health services. The development of the $1^{\text {st }}$ National Health Sector Strategic Plan (NHSSP-I, 1999-2004) was a follow-up to the Ministry of Health's (MoH) efforts to translate the policy objectives into an implementable programme. The strategic objectives of NHSSP-I are; (i) strengthening governance, (ii) improving resource allocation, (iii) decentralization of health services and management, (iv) shift of resources from curative to preventive and PHC services, (v) provision of autonomy to provincial and national hospitals and (vi) enhancing collaboration with stakeholders under a Sector Wide Approach (SWAp) modality [20].

The $2^{\text {nd }}$ National Health Sector Strategic Plan's (NHSSP II, 2005-2010) strategic objectives, to contribute to the reduction of health inequalities and to reverse the downward trend in health related impact and outcome indicators that had been noted during the implementation of the NHSSP-I. Its strategic objectives are; (i) increase equitable access to health services, (ii) improve quality and the responsiveness of services in the sector, (iii) improve the efficiency and effectiveness of service delivery, (iv) foster partnerships in improving health and delivering services, and (v) improve financing of the health sector [17].

\section{Health Sector of Kenya}

The health sector of Kenya comprises of three owner systems, with the Government running 41\%, the Nongovernmental organizations (NGOs) 15\%, and private businesses $43 \%$, which provide mainly curative health services and very few preventive services. The Government owns most of the hospitals, health centers, and dispensaries. On the other hand clinics and nursing homes are entirely in the hands of the private sector. The best-off Central Province has about twice the number of facilities per population as the worst-off provinces, such as, Nyanza and Western Valley. Central, Coast, and Eastern provinces have better ratios than the national average. In 2002 total number of health personnel was 59,000 of them 5,000 was doctors [59].

In Kenya, health services are provided through a network of over 5,000 health facilities countrywide. The public health sector consists of the following levels of health facilities [45]:

- national referral hospitals,

- provincial general hospitals,

- district hospitals, and

- health centers, and dispensaries.

National referral hospitals are at the apex of the health care system, which provide sophisticated diagnostic, therapeutic, and rehabilitative services. The two national referral hospitals in Kenya are Kenyatta National Hospital in Nairobi and Moi Referral and Teaching Hospital in Eldoret. The equivalent private referral hospitals are Nairobi Hospital and Aga Khan Hospital in Nairobi. They are centers of excellence and provide complex health care requiring more complex technology and highly skilled personnel [60].

Provincial hospitals form a secondary level of health care for their location. They provide services to a geographically well-defined area. They provide specialized care, involving skills and competence not available at district hospitals, which makes them the next level of referral after district hospitals. Their personnel include medical professionals, such as general surgeons, general medical physicians, paediatricians, general and specialized nurses, midwives, and public health staff. These hospitals provide medicine, obstetrics and gynaecology, accident and emergency services, ear, nose and throat (ENT) dental services, general surgery and anaesthesia, paediatrics, psychiatry, ophthalmology, dermatology, intensive care unit (ICU) and high dependency unit (HDU) services [17].

District hospitals provide very specialized care at the district level, maintain quality standards, and coordinate and control all district health activities. They are the first referral hospital and form an integral part of the district health system. These hospitals concentrate on the delivery of health care services and generate their own expenditure plans and budget requirements based on guidelines from headquarters through the provinces. At the district level, curative services are provided by district hospitals and mission hospitals. Public health services are managed by the District Health Management Team (DHMT) and Public Health Unit of the district hospitals. The DHMT and District Health Management Board (DHMB) provide management and supervision support to rural health facilities. District hospitals offer quality clinical care by a more skilled and competent staff than those of the health centers and dispensaries. They provide accident and emergency services, obstetrics and gynaecology, child health, medicine, surgery, including anaesthesia [9].

Health centers are staffed by midwives or nurses, clinical officers, and occasionally by doctors. They provide preventive and curative services, mostly adapted to local needs. They also provide a wider range of services, such as basic curative and preventive services for adults and children, as well as reproductive health services. They also provide minor surgical services such as incision and drainage. They augment their service coverage with outreach services, and refer severe and complicated conditions to the appropriate level, such as the district hospital [45].

The dispensaries are at the lowest level of the public health system and are the first point of contact with patients. But in some areas, health centers or even hospitals are effectively the first points of contact. They are staffed by enrolled nurses, public health technicians and medical assistants. The enrolled nurses provide antenatal care and treatment for simple medical problems during pregnancy such as anaemia, and occasionally conduct normal deliveries. Dispensaries provide wider coverage for preventive health measures, which is a primary goal of the health policy. At the sub-district level, both preventive and curative services are provided by the health centers as well as dispensaries and outreach services to the communities within the catchment areas [44].

Highly skilled physicians, nurses, administrators, and ancillary staff are critical to producing high-quality treatment and growth of hospital in Kenya [2].

\section{Malaria}


Malaria is a disease caused by parasites of the genus Plasmodium. According to Dr. Teresa Omwoyo, the National Health and Nutrition Coordinator for World Vision Kenya, malaria is the number one killer of children and women in Kenya [46]. Malaria is a serious and potentially fatal disease. Kenya is in the $5^{\text {th }}$ rank in a list of African region which account for $90 \%$ of malaria cases. Nigeria, Democratic Republic of Congo (DRC), Ethiopia and Tanzania make up the top four of the 19 country list [61]. About 30 million out of a population of 45 million (about 3 out of 4) Kenyans are at risk of malaria. Kenya has 63 malaria-endemic districts (out of total 72 districts) where $73 \%$ of country's population at risk of malaria. Among them 16 are in the highland malaria epidemic prone districts and the 8 arid and semi-arid districts have an unstable malaria epidemiological pattern [64].

\subsection{Statistics of Malaria in Kenya}

In Kenya, 1.5 million women become pregnant each year, and the majority live in malaria endemic areas. Pregnant women, children under-5 and the elderly are particularly vulnerable to serious infection of malaria due to their low immunity. Pregnant women who are suffering from malaria not only become anaemic but also are at risk of delivering babies with low birth weight.

Fetal complications of pregnant women may include low birth weight, abortion, stillbirth, prematurity, intrauterine growth retardation or congenital malaria infection. Pregnant women are 10 times more likely to contract malaria than non-gravid women. Pregnant women who contract malaria have a greater tendency to develop severe malaria [64].

Effectiveness of malaria in Kenya varies from region to region and the risk increase by altitude, rainfall patterns and temperature. Lake Victoria (Nyanza and the Western regions) endemic region (altitudes ranging from 0 to 1,300 $\mathrm{m}$ ) is high malaria transmission area for all year long and prevalence rate is $20-40 \%$. Coast area is another endemic region of malaria transmission all year long which prevalence less than $5 \%$. The vector life cycle is usually short and survival rates are high because of the suitable climatic conditions in these regions. Highland endemic region is the second malaria transmission area and prevalence rate is $0-20 \%$. Arid and semi-arid areas are of northern and south-eastern parts of the country which experience short periods of malaria transmission only during the rainfall seasons where prevalence is less than $5 \%$. Temperatures are usually high and water pools created during the rainy season provide breeding sites for the malaria vectors. Epidemic outbreaks with high morbidity rates happen due to the low immune status of the population when these areas are flooded. Malaria transmission in the Western Highlands of Kenya is seasonal and epidemics are experienced when long rains favor with minimum temperatures around $18^{\circ} \mathrm{C}$ and sustain vector breeding, results in increased intensity of malaria transmission and the epidemic becomes ten times greater than those experienced in regions where malaria occurs regularly. Central Highlands including Nairobi are of low risk area where little or no malaria transmission happens and prevalence rate is $0.1 \%$ [44].

In 2013, Kenya lost more than 46,000 people from malaria. According to World Health Organization (WHO) each year about 34,000 children under-5 die from malaria in Kenya (7,843 in Rift Valley, 5,878 in Eastern, 5,400 in Coast, 3,909 in Nyanza, 3,103 in Western, 389 in Central, 419 in Nairobi, and 141 in North-eastern). All four species of human Plasmodium: Plasmodium falciparum, Plasmodium malariae, Plasmodium ovale and Plasmodium vivax occur in Kenya and Plasmodium falciparum which causes the severest form of the disease accounts for $98.2 \%$ of all malaria infections, while $P$. malariae, $P$. ovale is $1.8 \%$ often occurring as mixed infections $[41,44]$.

About $30-50 \%$ of all outpatient morbidity and $20 \%$ of inpatient admissions in hospitals are due to malaria and about 170 million working days are lost annually due to malaria illness and each Kenyan family spends KSh 1,400 or more annually to treat malaria. It is estimated that malaria accounts for about $6 \%$ of all deaths and 20-25\% of deaths in children under- 5 have been certified to malaria in Kenya. Only 1 out of 10 children under-5 in the Lake-endemic region sleeps in a home that has been sprayed. Only 1 out of 50 dispensaries use the malaria rapid diagnostic tests and 2 out of 3 dispensaries do not diagnose malaria by blood tests [24].

Malaria is the reason of complications in pregnancy and can lead to early miscarriage. In Kenya out of 3 pregnant women living in the malaria endemic area around Lake Victoria does not use preventive malaria drugs. 2 out of 5 pregnant women in the Highlands endemic region do not sleep under ITN. 1 out of 7 women in areas where malaria is rampant does not seek antenatal care [44].

\subsection{Control of Malaria}

To combat malaria, in 2001, the Ministry of Health (MoH) through the National Malaria Control Programme (NMCP) developed the National Malaria Strategy (NMS) covering the period 2001-10, which emphasized the increased use of ITNs [26]. By the late 1990s, there was considerable evidence that the use of ITNs reduced childhood mortality in SSA [22]. Before 2001 mosquito net use was limited in Kenya. In 2002, Population Services International (PSI) Kenya received support from the UK Department for International Development to market partially subsidized ITNs across Kenya. The ITNs were priced at $\$ 4.7$ in urban areas and at $\$ 1.3$ in rural areas. In 2004, Kenya received a Global Fund grant to distribute 5 million long lasting insecticide nets (LLINs) free of charge to children under-5 [36].

Kenya has achieved a steady increase in household ownership of ITNs from 2004 to 2008. There have been tremendous gains in the ITN coverage rising from as low as $4.6 \%$ in 2003 to about $50.2 \%$ in 2008 for both pregnant women and children under-5, after a free mass ITN distribution but in the malarious regions ITNs rose to $60 \%$. In the highly malarious Nyanza and Western regions, household ownership of ITNs rose from 5.3\% and 2.4\% in 2003 to $76.5 \%$ and $71 \%$ respectively by $2008-09$. In 2002-08 periods, an estimated 16 million ITNs and LLINs were distributed in Kenya through various channels [21].

In collaboration with partners, the Government has developed the 10-year Kenyan National Malaria Strategy (KNMS) 2009-2017 which was launched in November 4, 2009. The goal of the National Malaria Strategy (NMS) is to reduce morbidity and mortality associated with malaria 
by $30 \%$ by 2009 and to maintain it to 2017 . More than $50 \%$ of pregnant women used ITN by the year 2008 for most provinces except Central and Rift Valley which had less $30 \%$ of pregnant women using ITN. There has been an overall increase in the coverage rate of ITN use by children under-5 (50\%) from 2003 and 2008 [64].

National malaria strategy recommended for 1 ITN for every 2 people but in malaria endemic areas there is 1 ITN to 5 Kenyans. Two out of 5 children under-5 in the malaria endemic Nyanza and Coast regions do not sleep under ITN. The current ITN ownership of 0.8 per household in Kenya is far from universal access defined as 2 nets per household. The main goal of the National Malaria Strategy (NMS) was to reduce the level of malaria infection and consequent death by 30\% by the year 2006 and to sustain that improved level of control to 2010.

To achieve the goal, the current $\mathrm{MoH}$ policy on malaria recommends several strategies as follows [17]:

- All pregnant women living in areas prone to malaria should have access to at least 2 free sulphadoxinepyrimethamine (SP) doses, or 2 Intermittent Prophylactic Treatment (IPT) doses.

- The policy also provides for personal protection to people at risk of malaria especially young children and pregnant women through increased access to ITN and IRS services.

- All fevers in children under-5 be presumptively treated as malaria with artemesinin combination treatment (ACT) and improved laboratory diagnosis, which is provided free of charge at Government and mission facilities.

- Improvement of epidemic preparedness and response.

- Application of cross-cutting strategies, such as, Information, Education and Communication (IEC) for Behavior Change Communication (BCC), Health Systems Strengthening (HSS) strategic actions, as well as effective monitoring and evaluation.

Pregnant women living in malaria endemic areas should get at least 2 doses of IPT, along with the prerequisite of effective case management for anaemia and malaria during pregnancy to reduce morbidity and mortality of both mother and child [39]. The proportion of women who received at least 2 doses of IPT rose from 4\% in 2002 to $24.5 \%$ in 2006 in sentinel districts and to $13 \%$ in all malaria endemic districts in 2007. In 2009, the Government vision of a Malaria-Free Kenya emerged as a result of the development of a multi-sector malaria control strategy 2009-2017 with clear and focused strategic approaches and objectives [17].

The National Malaria strategy recommended selective use of indoor residual house-spraying (IRS) in the early detection phase of epidemics. IRS is carried out in highland 16 malaria epidemic-prone districts. The strategy is to spray over $80 \%$ of the households in malaria epidemic-prone district to minimize transmission. The proportion of targeted IRS rose from $27.1 \%$ in 2005 to $63 \%$ in 2008. The intensity of IRS campaigns fluctuated due to inconsistent flow of financing. For example, in 2008, 63\% of the targeted houses were sprayed which covered protection to about 3.1 million people, but in 2009 , only $33 \%$ of the target was reached due to delays in donor fund disbursement. IRS is also affected due to poor quality control of pumps and insecticides [41].
Over the last 10 years, Kenya has made progress in malaria control. However, the country is still far from defeating the disease. The main reasons for this are poor knowledge of the disease and the lack of diagnostic equipment in health facilities, not sleeping under ITNs, lack of taking anti-malarial drugs during pregnancy. To roll back malaria, the Government must invest more in new initiatives and tools for fighting it even as it makes use of emerging epidemiological knowledge of the disease [64].

\subsection{Malaria Prevention}

Actually no malaria prevention treatment is $100 \%$ effective. Malaria risk can be reduced by avoiding mosquito bites by applying various techniques, such as, wearing long-sleeved shirts, sleeping under nets or in screened rooms, staying indoors or screened areas at dusk and using insect repellents and mosquito coils [61].

\subsection{Treatment Facilities of Malaria in Kenya}

If a pregnant woman becomes infected, the antimalarial and antiprotozoal drugs used to treat malaria and are safe for use during pregnancy for the mother and the fetus. Children need a shorter course for rapidly progressing to severe malaria. Children with severe malaria are more likely to present with hypoglycemia, seizures, severe anemia and sudden death, but they are much less likely to develop renal failure, pulmonary edema or jaundice. Cerebral malaria results in neurologic sequelae in 9-26\% of children. Children usually recover even from severe malaria much faster than the adults. If proper dosage is given, most antimalarial drugs are very effective and safe in children [64].

In Kenya 1 in 4 children with fever received some formulation of anti-malarial, only about 1 in 10 of these receives the treatment within 24 hours of fever. About 1 in 20 of children receives an appropriate first line antimalarial drug [44].

$P$. falciparum is resistant to chloroquine treatment. Primaquine is required in the treatment of $P$. ovale and $P$. vivax infection in order to eliminate the hypnozoites. Nonimmune pregnant women in endemic areas use the proper pharmacologic and non-pharmacologic prophylaxis. For an infected pregnant woman the anti-malarial and antiprotozoal drugs use are safe for both the mother and the fetus. In the sexual stage of the protozoan (the gametocyte) does not respond to most standard medications (e.g., chloroquine, quinine), but gametocytes eventually die and do not cause a threat to the individual's health [61].

Standard antimalarials, such as, chloroquine and antifolates (sulfadoxine pyrimethamine), are ineffective in many areas of Africa, because of increasing prevalence of drug resistance and a high likelihood of resistance development to new agents. Combination therapy is now becoming the standard of care for treatment of $P$. falciparum infection [57].

The treatment policy for malaria has changed since 1998 due to failing therapeutic efficacy from chloroquine to sulphadoxine-pyrimethamine (SP) and subsequently to the currently recommended artemisinin-based combination therapies (ACTs) in 2004. At present ACTs that are co-formulated are the best treatments for uncomplicated malaria and the efficacy of the treatments 
of malaria in Kenya [44]. It is strongly recommended that ACTs should include at least 3 days of treatment with an artemisinin derivative [64].

The commonly used confirmatory tests to detect the presences of malaria parasites in Kenya are microscopy or rapid diagnostic tests (RDTs). Microscopy is the standard method for parasitological diagnosis of malaria and is performed by examining a stained thick or thin blood smear for the presence of malaria parasites. RDTs are immunochromatographic simple tests based on detection of specific parasite antigens [44].

The first line treatment for uncomplicated malaria in Kenya is artemether-lumefantrine (AL) tablet containing $20 \mathrm{mg}$ of artemether and $120 \mathrm{mg}$ of lumefantrine. This is advised as a 6-dose schedule given over three days. The recommended second line treatment for uncomplicated malaria in Kenya is dihydroartemisinin-piperaquine (DHA-PPQ). This is currently available as a fixed-dose combination with adult tablets containing $40 \mathrm{mg}$ of dihydroartemisinin and $320 \mathrm{mg}$ of piperaquine and paediatric tablets containing $20 \mathrm{mg}$ dihydroartemisinin and $160 \mathrm{mg}$ of piperaquine. These are advised once daily for three days. The recommended treatments for severe malaria are parenteral quinine or parenteral artemisinins (artesunate or artemether). The preferred route of administration is the intravenous route [44].

\subsection{Knowledge Gap}

One out of 11 mothers in Kenya does not believe that malaria medicine can cure a child's fever. Children of the richest Kenyans under-5 are 5 times more likely to have their blood tests for malaria when they have a fever compared to children of the poorest. Out of 3 mothers in the semi-arid seasonal mothers believe that a child's fever should first be treated with herbs. Too many mothers do not believe that malaria medicine can cure a child's fever. More than half of the mothers in the Coast-endemic area have not heard, watched or read any information about the most common anti-malaria medicine [61].

Kinung'hi et al. [19] in a survey of very small amount of villagers of Muleba district of North-western Tanzania found that some people believe malaria can cause due to bedbugs $(0.6 \%)$, ticks $(1.6 \%)$, contaminated water $(15 \%)$, basking in sun-shine $(2.6 \%)$, working in the rains $(1 \%)$ and witchcraft $(0.4 \%)$ and similar results are found in other countries of SSA including Kenya. But most of them are confirm that malaria causes through the mosquito bites.

\subsection{Progress in Reduction of Malaria in Kenya}

The Global Fund, the GoK and the African Medical and Research Foundation signed in a grant agreement worth $\$ 80$ million to reduce malaria morbidity and mortality in Kenya by two-thirds by 2017 [64].

In recent years a remarkable progress has been made in malaria control in Kenya. This is due to widespread distribution of ITNs and LLINs and household spraying have helped large parts of the country become free from malaria [9].

Amount of pregnant women in malaria prevalence areas who receive two or more doses of IPTp during their pregnancy will have increased to $50 \%$. The household use of at least one ITN has increased to $70 \%$. The pregnant women sleeping under an ITN the previous night have increased to $60 \%$. About $95 \%$ of houses in malaria prevalence areas targeted for IRS have been sprayed. Antimalarial drug use also has increased in Kenya [44].

\section{Tuberculosis (TB) in Kenya}

Tuberculosis is caused by Mycobacterium tuberculosis also called Acid-Alcohol Fast Bacilli (AAFB) because of its staining properties. M. tuberculosis was discovered by Dr. Robert Koch in 1882. Transmission of this fatal disease is by droplet nuclei infection through coughing and sneezing. The incidence of TB is increased due to its association with HIV/AIDS, poverty, malnutrition and overcrowding [29].

Early symptoms of active TB disease are coughing up blood more than 2 weeks, unexplained weight loss, consistent low-grade fever, chills, haemoptysis, excessive night sweats, and loss of appetite. TB bacteria can remain in this latent state for months, years, and even decades without increasing in number and without signs and symptoms, or radiographic or making the person sick. This is called latent TB infection (LTBI). People who have latent TB infection do not get sick, never show any symptoms of TB and do not spread the bacteria to others. Only about $10 \%$ of people infected with M. tuberculosis ever develop tuberculosis disease [63].

Kenya is among the top 22 countries that collectively contribute to $80 \%$ of the world's TB cases and is ranked $13^{\text {th }}$, and has the $5^{\text {th }}$ highest burden in Africa according to the WHO Global Tuberculosis Report 2010. Kenya developed a national direct observation therapy (DOTS) strategy that was finalized in 1991. The DOT treatment success rate met WHO's target of 85\% in 2007 [65].

In Kenya the number of TB cases was 228 per 100,000 people in 2007 which is high in the world. Kenya reported more than 106,083 TB cases in 2010 with more than 4,000 deaths. WHO estimated that there were around 2,000 cases of multidrug-resistant TB (MDR TB) in Kenya in 2007 , although only $4.1 \%$ of these cases were diagnosed and notified. The annual budget for adult TB first-time treatment in Kenya is Ksh 300 million (150 for the required stock and 150 for buffer stock) and an extra Ksh 50 million pediatric TB treatment [48].

The TB programme has 12 operational zones (by sub dividing four of the largest provinces into 8) and 215 TB operational districts to facilitate TB control activities in Kenya. Most cases of TB in Kenya (80\%) are pulmonary. In Kenya mainly first-line drugs; isoniazid, rifampin, streptomycin, ethambutol and pyrazinamide are used to treat the TB. Most patients are in drug susceptibility of these medicines. The majority of the patients (66.5\%) were unemployed or self-employed in small businesses, with $79.4 \%$ earning less than $\$ 100$ per month. As a result they cannot take proper treatment due to financial crisis [35].

Both morbidity and mortality due to TB in Kenya is a major national health problem. TB affects all age groups, but has its greatest toll on those above 15 years of age. Epidemic HIV, poverty, and rapid urbanization led to increase of urban slums, prison congestion and limited access to general health care services are major causes of burden of TB disease. For the growing burden of TB in 
Kenya, the Ministry of Health formed the Division of Leprosy, Tuberculosis and Lung (DLTLD) to increase support in the followings [17]:

1. Strengthening of the human resource capacity at all levels of the DLTLD for effective coordination of TB control activities.

2. Decentralization of TB control services down to the community level to increase access to services.

3. Strengthening the collaboration between TB and HIV control programmes in order to promote delivery of integrated TB/HIV services, private-public partnerships to increase the number of private providers integrated into the $\mathrm{TB}$ service provider network.

4. Sustaining public education campaign coupled with health care worker training and support to promote early care seeking and adherence to treatment at community level and better TB case management by health care provider.

5. Sustained political commitment to increase human and financial resources and integrating TB control into the national health system.

6. Uninterrupted supply of quality assured drugs with reliable procurement and distribution systems.

Due to various attempts are taken by the DLTLD, the TB case notification rate (CNR) rose from 51 to 329 per 100,000 populations between 1987 and 2006. The treatment success rate has been a steadily increasing to a rate of $82 \%$ in the year 2006. Lack of emergence of drug resistant TB is a major challenge in the fight against TB. The WHO Global TB report for 2009 estimated that Kenya had approximately 132,000 new TB cases and an estimated 74,000 people died [62]. There are 2,000 treatment facilities and $900 \mathrm{~TB}$ diagnosis facilities throughout the country but they do not cater to drugresistant TB. In 2007, about 2,000 MDR TB cases were in Kenya, although only $4.1 \%$ of these individuals were diagnosed but the Government had no ability to treat them. In Kenya, about $20 \%$ of the 1.2 million HIV-positive individuals also have TB [55].

Kenya is the first country in SSA to have achieved the global targets for both case detection and treatment success and the country continues to treat more and more TB patients each year. But widespread co-infection with HIV (about $48 \%$ of new TB patients) makes TB diagnoses and treatment difficult. In Kenya more than $11 \%$ of all TB cases notified were children. MDR and childhood TB are the two main challenges the country [7].

\section{HIV/AIDS in Kenya}

Kenya is $4^{\text {th }}$ largest HIV epidemic country in the world. Out of the people 45 million about 1.6 million are living with HIV. About 820,000 women aged 15 and up, and 200,000 children aged 0-14 living with HIV. More than 1.1 million children have been orphaned by it and 80,000 Kenyan had died from AIDS related illnesses in 2012. About $80 \%$ of the AIDS related deaths occur among adults. In Kenya HIV affected all level of people, such as, infants, children, young people, women, men and elderly persons [56]. The first case of HIV in Kenya was detected in 1984, and by the mid 1990s it was one of the major causes of mortality in the country. Kenya's HIV prevalence peaked to $11 \%$ during the year 2000 and had been on the decline to $6.1 \%$ in 2012 due to an increase in awareness and education and the rapid scaling up of antiretroviral treatment (ART). Still many people are not being reached with HIV prevention and treatment services even with the steady increase in access of services [51,52].

HIV prevalence among urban adults is about $10 \%$ and rural adults are about 5.6\%. HIV prevalence in Nyanza province nearly doubles the national average. The estimated number of people living with HIV in Wajir is lowest 500, compared to a massive 140,600 in Homa Bay, 281 times higher [51].

Since 1995 HIV declined by about 40\% to reach approximately $6.7 \%$ in 2003 . Since then, the prevalence has remained relatively stable. The decline of the prevalence from 1995 to 2003 is partly attributed to high AIDS related mortality and the stabilization of the epidemic in the last 10 years is largely due to the rapid scale up of ART and reduction in the number of new infections that occurred during this period [52].

New infections among adults are more than $80 \%$ of the total new infections. The new infections among adults stabilized at an average of 93,000 annually over the last 5 years. Among children, new infections declined from about $20,000(18 \%)$ to $11,000(11 \%)$ annually over the same period [52].

HIV prevalence among men who have sex with men (MSM) in Kenya is almost three times (18.2 \%) that among the general population. In 2011, about $18.3 \%$ of people of inject drugs users (IGUs) in Kenya were living with HIV and most of them are in Nairobi and Mombasa. Use of condom and safe injecting practices are very low among this group and are responsible for high rates of HIV transmission [52]. Female sex workers and their clients have the highest reported HIV prevalence of any group in Kenya. About 29.3\% of them were living with HIV in 2011 but recently they are using condoms [50].

Girls in Kenya face discrimination in terms of access to education, employment and healthcare. They are forced into early marriage and often unable to negotiate safe sex. In 2012, about $6.9 \%$ of girls and women aged 15-24 were living compared with $4.2 \%$ of men. Young women are almost three times (3\%) more likely to be living with HIV than men $(1.1 \%)$ of the same age due to lack of knowledge of HIV infection among girls [32]. From the above statistics HIV prevalence in Kenya are given in Figure 1.

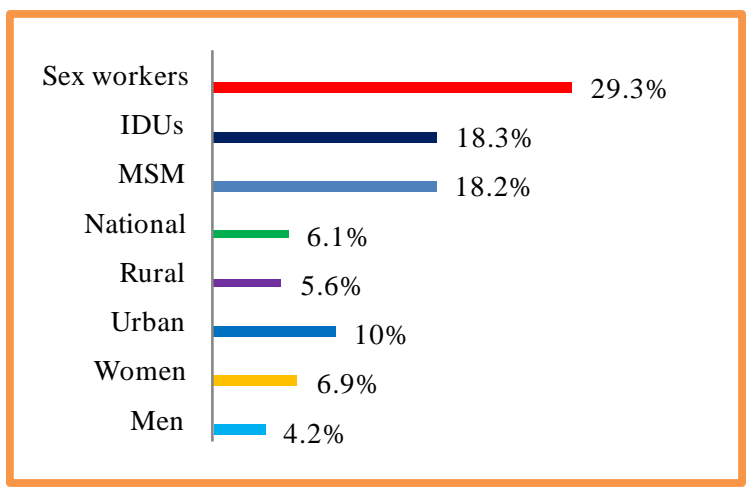

Figure 1. HIV prevalence rate (\%) in Kenya. Source: Prepared by author

About 55.3\% male and 46.6\% young people (aged 1524) have comprehensive knowledge of HIV in 2008-2012. Prevention among young people (aged 15-24) who use 
condom with multiple partners is $67.3 \%$ male and $37.1 \%$ female in 2008-2012 [51].

\subsection{HIV Testing Facilities in Kenya}

HIV testing facilities are increasing grammatically in Kenya. In 2000, there were just 3 voluntary counseling and testing (VCT) sites in Kenya but in 2010 the sites become more than 4000 . In 2008 , about 860,000 people were being tested annually for HIV and in 2013; this had increased to 6.4 million. From 2009 to 2013, the percentage of pregnant women tested for HIV increased from $68 \%$ to $92 \%$ [52]. VCT facilities are less among men than women in Kenya. In 2012, about $35.8 \%$ of men had a HIV test compared with $47.3 \%$ of women [31].

HIV testing rates among pregnant women have increased substantially. From 2009 to 2013, the percentage of pregnant women tested for HIV increased from $68 \%$ to $92 \%$ [52].

\subsection{Antiretroviral Therapy (ART) in Kenya}

In 2003, only 6,000 people living with HIV were given ART, but in 2013 this had increased to 656,000. In 2006 President of Kenya announced that ART would be provided for free in public hospitals and health centers. In 2009 the number of people receiving ART had significantly increased to 336,980 [52].

\subsection{Effect of Economy for HIV Infection}

Orphaned children are disadvantaged in schooling compared with children of non-HIV-infected parents. Fostered children also tend to be more undernourished than children of non-HIV-infected parents. Children of HIV infected parents are more likely to be underweight and wasted, less likely to be attending school, and less likely to receive medical advice and treatment when suffering from acute respiratory infections or diarrhea than children of non-HIV-infected parents [25].

AIDS also has an impact on labor productivity. Under the relatively controlled conditions of a Kericho tea estate, AIDS sufferers show significant declines in the production [10].

HIV infected persons compel to sell their household belongings for buying food and treatment. Sometimes HIV infected parents withdraw their children from school to provide care and engage them in child labor to maintain household expenditures. Sometimes women and children become the victims of sexual exploitation. As they have no other means except multiple sexual partnerships for supporting their families, which speeded HIV among the non-infected persons. When a woman's husband died the widow lose all of her property, and her sons possess all the properties. Those subject to physical or sexual violence are unable to escape abusive partnerships, because divorce would mean their losing all rights to property [23].

\subsection{HIV and TB Co-infection in Kenya}

Kenya is considered to have one of the highest coinfection prevalence rates of HIV and TB in the world. About $60 \%$ of people with TB are co-infected with HIV and the mortality rate attributed to $\mathrm{TB}$ in this group being above 130 per 100, 000. Kenya has a target of reducing
50\% TB deaths in HIV infected by 2015. The percentage of estimated HIV - positive incident TB cases that received treatment for both TB and HIV increased from $56.4 \%$ in 2011 to $82.8 \%$ [37,40].

\section{Infant and Child Health Care in Kenya}

Each year more than 10.8 million children under-5 die in the world mainly from preventable diseases that could be treated effectively. 42 countries account for $90 \%$ of child deaths while 6 countries account for $50 \%$ of the deaths [4]. The lives of an estimated 6 million children could be saved each year if antibiotics for pneumonia and oral rehydration therapy for diarrhea were universally available [14]. Only in 2012 more than 100,000 children died before their fifth birthday, largely due to preventable causes. About 13,000 new HIV infections occurred among children and $62 \%$ of children living with HIV did not access life-saving antiretroviral drugs [53].

Kenya Demographic and Health Survey (KDHS) in 2003 expressed that 1 in every 9 children born dies before age five, mainly of acute respiratory infection, diarrhoea, measles, malaria, and malnutrition [58]. The Government of Kenyan (GoK) is also committed to the achievement of Millennium Development Goal number 4: reducing the infant and under-5 mortality rates to 21 and 32 per 1,000 childbirths respectively by the year 2015 [16].

The mortality rate of children under- 5 has been reduced by 36\% between the 2003 and 2008-09 in Kenya from 115 to 74 deaths per 1,000 live births and the infant mortality rate has been reduced with $32 \%$ from 77 deaths per 1,000 in 2003 to 52 deaths per 1,000 in the 2008-09 [15].

\section{Financing in Health Sector}

According to the 2001-2002 National Health Accounts (NHA), Kenya spends $5.1 \%$ of its GDP on health. The health budget has grown significantly from Ksh15.2 billion in Fiscal 2001-02 to Ksh34.4 billion in Fiscal year 2008-09. There are three major sources of financing for health services in Kenya: the government (central and local) contributes $30 \%$, with households paying 51\% out of pocket and donors (international and domestic) 16\%; the legal National Hospital Insurance Fund (NHIF) and other private insurers and sources contribute the rest [42].

Donor funding for the health sector as a share of the total budget has increased from 8\% in Fiscal 1994-95 to $16 \%$ in Fiscal 2001-02. Major donors include Japan, the US, the UK, and the European Commission [38].

Most of the people in Kenya have a high knowledge about HIV/AIDS and are aware of the prevention of this disease (98\% among women and 99\% among men) but this knowledge is lower among the people aged 15-19 years. Health care has also improved in Kenya. Total expenditure on healthcare increased from Ksh 17.6 billion (\$219 million) in 2005 to Ksh 22.3 billion (\$277.5 million) in 2007, and between 2004 and 2008 the number of health institutions increased by $19.8 \%$. In 2008, the Government began providing free antiretroviral drugs (ARVs) to HIVpositive people and medical care is now free for under-5 children [12]. 
In 2012, use of improved drinking water sources in Kenya is $60.9 \%$ (82.7\% in urban and 54\% in rural areas), use of improved sanitation facilities is $29.4 \%$ (31.1\% in urban and $28.8 \%$ in rural areas). Immunization coverage in 2012 is as follows: BCG 84\%, DPT1-89\%, DPT3-83\%, polio3- 82\%, MCV 93\%, HepB3-83\%, Hib3-83\%, tetanus (newborns) 73\%. Other health activities in Kenya are; care seeking for suspected pneumonia 55.9\%, antibiotic treatment for suspected pneumonia 49.6\%, diarrhoea treatment with oral rehydration saline (ORS) 38.8\%, antimalarial treatment among feverish children $32.2 \%$, malaria treatment of the children sleeping under Insecticide Treated Nets (ITNs) 46.7\%, malaria treatment of the households with at least one ITN 55.7\% [51].

The national TB program of Kenya requires an annual budget of about $\$ 62$ million. The Government only allocates about $\$ 6$ million. In 2010, the program received about \$21.5 million of which only \$7.4 million [48].

\section{Health Management System}

Management of healthcare at the district level is headed by a District Medical Officer of Health (DMOH) appointed by the Ministry of Health. The $\mathrm{DMOH}$ is supported by a District Health Management Board (DHMB) comprising officials appointed by the $\mathrm{MoH}$ and from local areas, and a professional unit, the District Health Management Team (DHMT). The DHMT prepares technical advisories and the District Health Plan in consultation with local health actors and the DHMB [33].

\subsection{Opportunities of Kenya}

Progressive rights-based constitution and clearly expressed Government strategies are as follows [49]:

- Strong health partnerships which provide additional resources for addressing health challenges.

- Robust private sector, economy and civil society strengthen service delivery capacity.

- Regional and sub-regional integration provides opportunities for harmonization of cross-borders.

\subsubsection{Challenges of Kenya}

High burden of communicable diseases and a growing burden of non-communicable diseases are as follows [49]:

- Weak health systems characterized by inadequate health infrastructure, human resources and other health care inputs exacerbating distribution inequalities and health services utilization.

- Persistent challenges in procurement, financial and information management to support health services decentralization.

- Lack of comprehensive approach to intervention in some key areas.

\subsubsection{Development in Health Sector in Kenya}

Before white man came to Kenya the people used herbs, animal parts, divination and others for treatment of diseases. After coming of the Christian missionaries and the colonial settlers' modern medicine was introduced and established mission hospitals and clinics in Kenya. After independence the medical sector has developed with modern technologies and medicines [34].
There are indications of ongoing improvements in the health status for infants and under-5. According to latest Kenya Demographic Health Survey (KDHS) [15], infant and under-5 mortality improved from 77 and 115 to 52 and 74 per 1,000 live births respectively. Maternal and neonatal mortality, on the other hand, had shown stagnation since 1993. As a result, the country is not on track to meet its health MDG targets (4, 5 and 6). Furthermore, there are wide disparities in health status across the country, closely linked to underlying socioeconomic, gender and geographical disparities. The country's burden of communicable diseases remains high. HIV/AIDS is responsible for up to $29.3 \%$ of all deaths, and $24.2 \%$ of all disability in the country. Other causes of death include conditions during and just after birth (9\% of deaths), respiratory (chest) infections including tuberculosis (14.4\% of deaths), diarrhoeal diseases (6\% of deaths), malaria (5.8\% of deaths), stroke (3.3\% of deaths), heart attacks (2.8\% of deaths), road traffic accidents $(1.9 \%$ of deaths), and violence (1.6\% of deaths).

Many interventions have been introduced, particularly since 2004, to address the high burden due to communicable diseases. As a result, investments in HIV, malaria, tuberculosis control, maternal and child health have been made. Additionally, investments in risk factors to health such as tobacco and alcohol use are being prioritized. These investments are complemented by investments in other health related sectors, such as in nutrition, access to safe water, education, roads, and others, to contribute to the overall improvement in health seen [15].

Kenya has recently experienced outbreaks of new/reemerging conditions such as polio and viral haemorrhagic fevers (e.g., dengue). Neglected tropical diseases such as lymphatic filariasis also remain a burden. Investments made since 2004 are now paying dividend [43].

Malaria incidence and prevalence has significantly declined, as confirmed through 2010 community survey results, which showed a reduction of malaria parasitemia in the lake endemic areas among children under- 5 by over $80 \%$ to $27 \%$ between 2002 and 2010 as a result in part of increased ITN coverage. Following the 2011 campaign over $65 \%$ of the households in both endemic and epidemic malaria achieved universal coverage, i.e., at least one net for every two people in a household [44].

HIV prevalence declined from its peak of above $10 \%$ in early 2003 to $6.3 \%$ in 2008 with a ratio of 2:1 for women to men. Furthermore, nearly $78 \%$ of those in need of ART are now accessing it [62].

Estimated TB incidence has also come down from the peak of 450 cases per 100,000 populations in 2005 to 200 in 2010. TB prevalence remains high at 319 per 100,000, but the country has managed to maintain its achievement of $70 \%$ case detection rate and $85 \%$ treatment success rate since 2006 [66].

Non-communicable disease burden is on the rise; diabetes prevalence is estimated at $3.3 \%$, and $13 \%$ of school-age children aged 13-15 years are active cigarette smokers. The country has ratified the Framework Convention on Tobacco Control (FCTC) and is party to International Health Regulation (IHR) 2005. Sanitationrelated illnesses like cholera remain a challenge in 
selected areas where adequate sanitation coverage remains low [43].

\section{Recommendations}

To develop the health sector of Kenya adequate number of high skilled and experienced employees must be employed continuously, discourage ineffective recruitment, encourage monitoring of doctors and nurses, and ensure that performance and practice standards are met to enhance service quality provision. Medication services, patient satisfaction, good relationship between medical providers and patients have to give priority to improve the quality of hospital. Modern health services are needed with very low costs or free of cost to make the healthy nation in Kenya. Government of Kenya must increase budget in the health sector and provide sufficient free medical services to the poor. Various national and international NGOs and social conscious organizations must contribute to make the health sector in a standard form.

\section{Concluding Remarks}

In this study we have discussed the health situation of Kenya. It is a developing country in the SSA. Childhood vaccination has to be increased in the country to reduce child morbidity and mortality rates. Infant and child mortality rate must be reduced to make a healthy nation in future. Every year a large number of Kenyans die due to curable and preventable diseases. Government must try to reduce poverty from the country. So the people can spend sufficient amount of money to take the proper treatment and can take balance diet to prevent diseases. In the study we have observed that health care facilities in Kenya are not sufficient in the health sector. Social consciousness must be increased to prevent TB, malaria, HIV and some other infectious diseases. We hope the article is relevant topics from the public health aspects of the common people.

\section{References}

[1] Abuja +12, "Shaping the Future of Health in Africa, Special Summit of the African Union on HIV/AIDS, Tuberculosis and Malaria”, Nigeria. 2013.

[2] Argote, L., "Organizational Learning: Creating, Retaining, and Transferring Knowledge”. New York: Springer. 2000.

[3] Black, R.E., Cousens, S., Johnson, H.L., Lawn, J.E., Rudan, I., Bassani, D.G., Jha, P., Campbell, H., Walker, C.F., Cibulskis, R., "Global, Regional, and National Causes of Child Mortality in 2008: A Systematic Analysis”. Lancet, 375 (9730): 1969-1987. 2010.

[4] Black, R., Morris, S. and Bryce, J., "Where and Why are 10 million Children Dying Every Year?” Lancet, 361: 2226-2234. 2003.

[5] Central Bureau of Statistics (CBS), "Kenya Demographic and Health Survey”, 2003, Central Bureau of Statistics, Nairobi, July 2004.

[6] CBS, MoH \& ORC Macro, "Kenya Demographic and Health Survey 2003”. Central Bureau of Statistics (CBS) [Kenya], Ministry of Health (MOH) [Kenya], and ORC Macro. Calverton, Maryland, USA. 2004.

[7] Civil Society UNGASS TB/HIV Country Report Kenya, "National Empowerment Network of People Living with
HIV/AIDS in Kenya (NEPHAK) \& Network of Men Living with HIV/AIDS in Kenya (NETMA+)”. 2010.

[8] Country Profile, "Republic of Kenya, African Union”. 2013.

[9] Country Profile, "Republic of Kenya, African Union”. 2014.

[10] Fox, M.P., Rosen, S., Macleod, W.B., Wasunna, M., Bii, M., Foglia,G. and Simon, J.L., "The Impact of HIV/AIDS on Labour Productivity in Kenya”, Tropical Medicine and International Health. 2004. 9 (3): 318-324.

[11] GoK, “Agricultural Sector Development Strategy”, 2010-2020. 2010.

[12] Hussein, A., "Response to Children Affected by HIV and AIDS in Kenya”. Irish Aid. 2008.

[13] IMF, “Kenya: Poverty Reduction Strategy Paper”. January 2005, IMF Country Report No. 05/11, International Monetary Fund, Washington DC. 2005.

[14] Jones, G., Steketee, R.W., Black, R.E., Bhutta, Z.A. and Morris S.S., "How Many Child Deaths Can We Prevent This Year?" The Bellagio Child Survival Study Group. Lancet. 2003. 362: 65-71.

[15] Kenya Demographic Health Survey (KDHS), "2003 and 2008-09”. 2009.

[16] Kenya Global Week of Action, “Saving Children’s Lives through Ensuring Equal Access to Quality Health Services”, Kenya. 2014.

[17] Kenya Health Policy Framework 1994-2010, “Analysis of Performance, Analytical Review of Health Progress, and Systems Performance”, Kenya. 2010.

[18] Kenya Nutrition Profile, "Food and Nutrition Division”, FAO, 2005.

[19] Kinung'hi, S.M., Mashauri, F., Mwanga, J.R., Nnko, S.E., Kaatano, G.M., Malima, R., Kishamawe, C., Magesa, S. and Mboera, L.E.G., "Knowledge, Attitudes and Practices about Malaria among Communities: Comparing Epidemic and Nonepidemic Prone Communities of Muleba District”, North-western Tanzania, BMC Public Health, 10: 395. 2014.

[20] Kizito, L., Kariuki, J., Muthami, L., Mutai, J., Ojakaa, D., Agina, O., Okoth, J., Muleshe, S., Bwonya, J. and Wasunna, M.K., "National Study to Review existing Policy Documents and Identification of upcoming Priority National Health Policy issues in East African Community Partner States: Kenya Country Report”, Kenya. 2008.

[21] KNBS and ICF Macro, "Kenya Demographic and Health Survey 2008-09”. Kenya National Bureau of Statistics (KNBS) and ICF Macro. 2010.

[22] Lengeler, C., "Insecticide-treated Bed Nets and Curtains for Preventing Malaria, Cochrane Collaboration”, John Wiley \& Sons, Ltd. 2004.

[23] Millennium Development Goals in Kenya, "Needs \& Costs”, the Ministry of Planning and National Development, Courtesy of the UNDP, Kenya, and the Government of Finland. 2005.

[24] Ministry of Public Health and Sanitation (MoPHS), "Kenya Malaria Programme Performance Review 2009”. Nairobi. 2009.

[25] Mishra, V., Arnold, F., Otieno, F., Cross, A. and Hong, R., "Education and Nutritional Status of Orphans and Children of HIV-Infected Parents in Kenya”, DHS Working Paper 2005 No. 4, United States Agency for International Development (USAID) Demographic and Health Research. 2005.

[26] MoH, “National Malaria Strategy 2001-2010”, Ministry of Health (MOH), Kenya. 2001.

[27] Mohajan, H.K., "Poverty and Economic Development of Kenya”, International Journal of Information Technology and Business Management, 18 (1): 72-82. 2013.

[28] Mohajan, H.K., "Food and Nutrition Scenario of Kenya", American Journal of Food and Nutrition, 2 (2): 28-38. 2014a.

[29] Mohajan, H.K., "Tuberculosis is a Fatal Disease among Some Developing Countries of the World”, (Unpublished Manuscript). 2014b.

[30] Mwabu, G., "Health Development in Africa”. Economic Research Papers Series (38), The African Development Bank Group: Abidjan, Cote d'Ivoire. 1998.

[31] NASCOP, "Kenya National Guidelines for HIV Prevention and Management of People Who Use Drugs (PWUD)”, Kenya. 2013.

[32] National AIDS/STI Control Programme, NASCOP, "Kenya AIDS Indicator Survey 2012”, Kenya. 2012.

[33] Ndavi, P.M., Ogola, S., Kizito, P.M. and Johnson, K. "Decentralizing Kenya's Health Management System: An Evaluation”. Kenya Working Papers No. 1. Calverton, Maryland, USA: Macro International Inc. 2009. 
[34] Ndetei, D.M., Mbwayo, A.W., Mutiso, V.N., Khasakhala, L.I. and Chege, P.M., "Traditional Healers and their Provision of Mental Health Services in Cosmopolitan Informal Settlements in Nairobi”, Kenya, African Technology Policy Studies (ATPS) Working Paper No. 67. 2013.

[35] Ndung'u, P.M., Kariuki, S., Ng'ang'a, Z. and Revathi, G., "Resistance Patterns of Mycobacterium Tuberculosis Isolates from Pulmonary Tuberculosis Patients in Nairobi”, Journal of Infection in Developing Countries, 6 (1): 33-39. 2012.

[36] Noor, A.M., Amin, A.A., Akhwale, W.S. and Snow, R.W., "Increasing Coverage and Decreasing Inequity in Insecticidetreated Bed Net use among Rural Kenyan Children”. PLoS Medicine, 4 (8), e255: 1341-1347. 2007.

[37] Nyamogoba, H.D.N., Mbuthia, G., Mining, S., Kikuvi, G., Biegon, R., Mpoke, S., Menya, D. and Waiyaki, P., "HIV Co-infection with Tuberculous and Non-Tuberculous Mycobacteria in Western Kenya: Challenges in the Diagnosis and Management”, African Health Sciences 12 (3): 305-311. 2012.

[38] OECD, "Development Database on Aid from DAC Members". Paris: OECD/DAC. 2006.

[39] Olum, G.H., "Conflict of Interest within the Kenya Country Coordinating Mechanism”, Kenya. 2007.

[40] Padmapriyadarsini, C., Narendran, G. and Swaminathan, S., "Diagnosis \& Treatment of Tuberculosis in HIV Co-infected Patients”, Indian Journal of Medical Research, 134: 850-865. 2011.

[41] Pathania, V., "The Impact of Malaria Control on Infant Mortality in Kenya”, Economic Development and Cultural Change, 62(3): 459-487. 2014

[42] Republic of Kenya, "Budget Speech for the Fiscal Year 2007/2008”. Nairobi: Ministry of Finance. 2008. Web: http://www.treasury.go.ke/downloads/budget_speech_2007.pdf

[43] Republic of Kenya, "Clinical Guidelines for Management and Referral of Common Conditions at Level 2-3: Primary Care", Clinical Management and Referral Guidelines-III, WHO. 2009.

[44] Republic of Kenya, "National Guidelines for the Diagnosis, Treatment and Prevention of Malaria in Kenya”, Ministry of Public Health and Sanitation \& Ministry of Medical Services. 2010.

[45] Republic of Kenya, "National Food and Nutrition Security Policy", Latest draft, June 2011.

[46] Siema, P., "Malaria Largest Killer of Children in Kenya", World Vision Kenya, Communications. 2014.

[47] Tam, J.L.M., "Examining the Dynamics of Consumer Expectations in Chinese Technologies", Journal of Marketing, 66 (3): 98-111. 2005.
[48] Tuberculosis Funding Situation in Kenya, "Health Policy Paper", Kenya. 2012.

[49] Turin, D.R., "Health Care Utilization in the Kenyan Health System: Challenges and Opportunities", The International Student Journal: 1-3. 2010.

[50] UNAIDS, "Sex Workers Advocate for Access to Health Care and Legal Services”, 12 March 2012.

[51] United Nations General Assembly Special Session (UNGASS), "Country Report-Kenya". United Nations General Assembly Special Session on HIV and AIDS. 2012.

[52] UNGASS, "Kenya AIDS Response Progress Report 2014: Progress towards Zero", Kenya. 2014

[53] UNICEF, "Committing to Child Survival: A Promise Renewed", Progress Report 2013. 2013.

[54] UN \& MPND, "Millennium Development Goals: Progress Report for Kenya". United Nations Office in Nairobi, Ministry of Planning and National Development of the Government of Kenya. 2003.

[55] USAID, “Tuberculosis Profile”. 2009. Web: http://www.itnhealth.net/medicalfiles/cme_series/module-004Havard/hiv_tb/assets/reports/USAID-kenya-2009.pdf.

[56] USAID, “Kenya HIV/AIDS Fact Sheet”, Kenya. 2013.

[57] US Food and Drug Administration, FDA, "Approves Coartem Tablets to Treat Malaria”. US Food and Drug Administration (FDA). 2009.

[58] Wamae, A., Kichamu, G., Kundu, F. and Muhunzu, I., "Child Health Services in Kenya”, Kenya Working Papers No. 2. Calverton, Maryland, USA: Macro International Inc. 2009.

[59] Wamai, R.G., "The Kenya Health System-Analysis of the Situation and Enduring Challenges", International Medical Community, JMAJ, 52 (2): 134-140. 2009.

[60] Wanjau, K.N., Muiruri, B.W. and Ayodo, E., "Factors Affecting Provision of Service Quality in the Public Health Sector: A Case of Kenyatta National Hospital", International Journal of Humanities and Social Science, 2 (13): 114-125. 2012.

[61] WHO, “World Malaria Report 2008”. Geneva. 2008.

[62] WHO, "Global Tuberculosis Control: Epidemiology, Strategy, Financing: WHO Report 2009”, Geneva. 2009a.

[63] WHO, “A Brief History of Tuberculosis Control in Kenya”. WHO Press, Geneva. 2009b.

[64] WHO, "Guidelines for the Treatment of Malaria", $2^{\text {nd }}$ Edition, WHO-GMP Geneva. 2010a.

[65] WHO, "Global Tuberculosis Control 2010: The Global Burden of TB”. WHO Press, Geneva. 2010b.

[66] WHO, “The Country Cooperation Strategy Briefs”, Kenya. 2013. 